Basic Biology of Aging at the University of Michigan in Ann Arbor. "It's a single genotype, and it's a weird genotype," he says. For instance, C57BL/6 mice often get serious skin problems in middle age and must be euthanized for humane reasons. They also have high incidences of eye abnormalities, acquired deafness, metabolic complications and low bone densities-all strain-specific issues that can present problems for studies of aging and longevity. So, separate from validating new assays for assessing aging in mice, Miller and his colleagues have developed a new mouse population to lessen the reliance on inbred mouse stocks for experimental analyses of aging.

The UM-HET3 mice are the product of a fourway crossbreeding scheme in which the mice are all of the progeny of four different inbred grandparental strains (one of which is C57BL/6). Importantly, this creates genetic differences

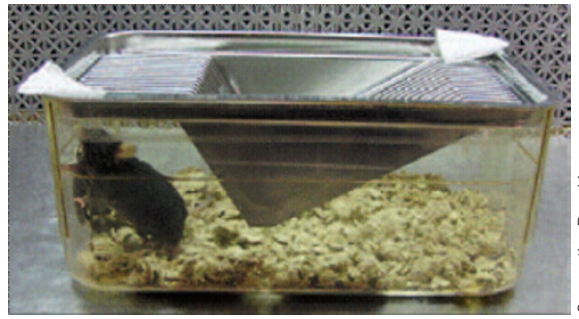

Sniff test: Urinary proteins show olfaction acuity.

among the mice-analogous to the genetic differences seen in human populations-and thus variation in their genetic predisposition for various diseases linked to aging. "We humans are extremely heterogeneous genetically," says Felipe Sierra, director of the NIA's Division of Aging Biology in Bethesda, Maryland. "Before we go to human beings, we have to see if a particular treatment works in a genetically heterogeneous background [in mice]."

The NIA has adopted the UM-HET3 mice as the standard for its Interventions Testing Program, which is evaluating candidate drugs such as antioxidants and anti-inflammatory agents that could delay rates of aging. Currently, the program leaders, who include Miller, are screening five compounds per year at three research centers across the US. As a first pass, they are simply looking at lifespan. But soon, many researchers hope, they will also be using more sensitive assays of age-related health, such as Pistell's T-maze or Austad's olfactory test.

"There's this consensus that longevity is not enough. We need to look at other measures of health," says Nathan LeBrasseur, director of the Mayo Clinic's Healthspan Assessment Laboratory. "This is really kind of a new wave in thinking about research."

Elie Dolgin

\title{
Sequencing of superagers offers drug targets, but fewer than hoped
}

At the age of 107 , Irving Kahn is older than commercial television, the theory of relativity and five US states. A money manager by trade, Kahn has outlived all but one of the companies of the original Dow Jones Industrial Index-and has done so in remarkable health. Kahn is still active today as chairman of a New York-based investment firm he founded with his sons, and he shows no signs of common age-related disabilities such as Alzheimer's or cardiovascular disease. It's a trait that runs in the family_each of his three siblings also lived past the age of 100 .

This remarkable health and longevity has led scientists to study the genetic makeup of centenarians like Kahn in the hope of discovering targets for the development of drugs that might someday be given to people without such genetic good fortune. The goal is to pharmacologically emulate features of these socalled 'superagers'. However, the pace of discovery has been slower than originally anticipated, owing to the byzantine nature of the aging process.

One of the best-studied genes affecting longevity encodes the cholesteryl ester transfer protein (CETP), an enzyme that helps turn 'good' cholesterol into 'bad' cholesterol. Ten years ago, a team led by Nir Barzilai, director of the Institute for Aging Research at the Albert Einstein College of Medicine in New York, discovered that Ashkenazi Jews with family histories of exceptional longevity were two to three times more likely to carry a genetic mutation that lowered CETP production compared to age-matched controls (JAMA 15, 2030-2040, 2003). A follow-up study found that lower CETP levels seemed to aid preservation of cognitive function and reduce the risk of Alzheimer's disease in this group, suggesting that drugs that block CETP could achieve the same effect (JAMA 2, 150-158, 2010).

"This is an example of where you come from this very unique, rare population of centenarians and find protective mechanism for drugs," says Barzilai, who consults for Merck, one of the two big pharma firms with CETP inhibitors now in late-stage clinical development.

\section{Outfoxed}

Studies involving centenarians and nonagenerians have also linked particular variants of the human forkhead box 03A (FOXO3A) gene and the apolipoprotein $\mathrm{E}(A P O E)$ gene with longevity traits. However, how FOXO3A and ApoE are regulated, their mechanisms of action and their functions are still largely unknown. Beyond these examples, the number of validated gene targets are few and far between.

Thomas Perls, director of the New England Centenarian Study at the Boston University School of Medicine, chalks that up to the genetic complexity that underscores healthy aging. "Getting to these extreme ages is not explained by a relatively few genetic variants with powerful effects," he says. "But rather it's on the score of hundreds of variants with relatively modest effects."

Last year, Perls and his colleagues published a controversial paper in PLoS One (7, e29848, 2012) that described a group of 281 single nucleotide polymorphisms that could be used to discriminate between centenarians and control populations. (A version of the work was previously published in Science but later retracted for technical errors. Perls stands by the main findings.) Some of these less common longevity-associated genes could now be interrogated for drug development, once their functions are worked out. "This kind of search may find targets that lower risk for some age-related diseases, and that wouldn't be too bad," says Evan Hadley, director of the division of geriatrics and clinical gerontology at the US National Institute on Aging in Bethesda, Maryland.

For now, experts agree, many more genomes still need to be sequenced to find more clues into exceptional longevity. There's even a $\$ 10$ million challenge from the X-Prize Foundation to spur scientists to develop better sequencing methods for centenarians (see Nat. Med. 17, 1329, 2011). And despite the dearth of drug leads, the genetics field remains enthusiastic. "I think we're in a very exciting phase in the study of exceptional longevity," Perls says. "People are looking fast and furiously for these protective variants that slow the rate of aging and decrease your chance of getting agerelated diseases."

Kevin Jiang 